\title{
Fast solidification kinetics of parts of bituminous binders
}

\author{
Hartmut R. Fischer · Bert Dillingh • \\ Bastiaan Ingenhut
}

Received: 26 March 2015/ Accepted: 28 September 2015/Published online: 12 October 2015

(C) RILEM 2015

\begin{abstract}
A quantification of the kinetics of formation of the micro-phases in bitumen during cooling as it occurs in applications, e.g. a road pavement will enable a better prediction of the performance of asphalt in models and an understanding of functional properties and their development in time. This microphase formation is part of the process of physical ageing (hardening) and starts almost immediately after or instantaneously during cooling. The earlier proposed process of liquid liquid demixing within bitumen upon cooling is followed by a, at the beginning homogeneously nucleated and three-dimensional crystallization, which changes in the course of crystallization to 2-dimensional crystal growth and mixed nucleation, both homogeneous and heterogeneous.
\end{abstract}

Keywords Bitumen - Solidification - Kinetics · AFM

\section{Introduction}

Bituminous binders undergo changes in properties with time, even in the course of annealing, storage and/ or use at room temperature- $\mathrm{a}$ phenomenon long

H. R. Fischer $(\bowtie) \cdot$ B. Dillingh · B. Ingenhut TNO Technical Sciences, De Rondom 1, 5612 AP Eindhoven, The Netherlands e-mail: hartmut.fischer@tno.nl known from practice and often called ageing. The underlying microstructural changes may be connected to two different processes occurring in parallel: (1) chemical reactions resulting in irreversible processes e.g. UV-irradiation assisted oxidation; leading to an increase and/or decrease in molecular size of single components of the bitumen and (2) physical aging or reversible processes such as re-arrangements of molecular aggregates, aggregation of molecules and crystallization of components within the mixture of compounds within the bitumen [1]. Both processes lead to an increase in hardness or to stiffening in time, the first one in a rather long time-scale (years). The second process is usually much faster (minutes up to several months).

The study of the process of physical hardening during extended storage at isothermal conditions dates back to 1936 when a report by Traxler and Schweyer [2] was published. Different asphalt binders were investigated and a substantial increase in viscosity with time of storage at room temperature was observed. The phenomenon was called in this time "age hardening". In a subsequent study, the process of increasing viscosity during isothermal storage was explained by the structural sol-gel transition [3] according to the micellar model of bitumen structuring [4].

Increase of the stiffness of several asphalt binders after isothermal storage was found in a later investigation by Brown et al. [5]. This process was called "steric hardening". The effect was explained by the 
collapse of the molecular free volume at low temperature, an isothermal sol-gel transition and wax crystallization. The term "physical hardening" of asphalt binders was first used by Blokker and Van Hoorn [6] who explained the process by wax crystallization and asphaltene aggregation.

In the current view, physical hardening which may be also termed as "spontaneous hardening" is connected with the establishment of (new) interactions between polar and apolar components leading to the formation of discrete crystalline domains as observed earlier. This process takes days to weeks if bitumen is stored at room temperature $[5,7,8]$ respectively at low temperatures, but still above $T_{\mathrm{g}}$ [9-12] and is basically a thermally activated process. Hardening effects during the cooling of a bitumen melt are also discussed by Masson et al. [8]. In this study, a four stage ordering within bitumen upon cooling from the melt was suggested. In the first stage, all fractions order rapidly into a weakly organized phase. In the second stage, low molecular weight saturated segments crystallize.

In the third stage, high molecular weight saturated segments crystallize. In the fourth stage, resins and asphaltenes order into a mesophase. The third and fourth stages are responsible for the room-temperature (steric) hardening of bitumen. The formation of microstructures during cooling down from an isotropic liquid as initial condition at high temperatures as well as the gradually melting during heating from a two phase structure show good agreement with the thermal signals recorded by differential scanning calorimetry (DSC) in a recent study [13].

It is certainly of interest to quantify the kinetics of the formation of the micro-phases during cooling of bitumen as it occurs in the applications of bitumen, e.g. a road pavement, to better predict performance of asphalt in models and to understand the functional properties and their development.

\subsection{Experimental part}

The bituminous binders used in this study are labelled Q8 70-100 and Total 70-100 and were supplied by Kuweit Petroleum Research \& Development (Rotterdam, Netherlands) and Latexfalt B. V (Koudekerk, The Netherlands). The first bitumen was derived from Kuweit Crude Export. The penetration (PEN) value was 82 , the softening point $45.8{ }^{\circ} \mathrm{C}$ and the Fraass breaking point $-15^{\circ} \mathrm{C}$. The content of SARA fractions is as follows: Saturates: $3.8 \%$, Aromatics: $59.6 \%$, Resins: $22.2 \%$, Asphaltenes: $14.6 \%$. The other bitumen had similar properties with a content of SARA fractions is as follows: Saturates: $5.9 \%$, Aromatics: $29.5 \%$, Resins: $47.0 \%$, Asphaltenes: $17.6 \%$. Bitumen films were prepared by the application of a bead of bitumen onto a steel disk, followed by a heating stage at $120{ }^{\circ} \mathrm{C}$ for $1-2 \mathrm{~min}$ in an oven to ensure a low viscosity of the binder. The resulting film thickness was about $300 \mu \mathrm{m}$. Prior to the AFM experiments, the sample was quenched with a rate of $30 \mathrm{~K} / \mathrm{min}$ to $60{ }^{\circ} \mathrm{C}$. Atomic Force Microscopy observations were performed on a NanoSurf Easyscan 2 apparatus operated in Tapping ${ }^{\circledR}$ mode combined with a Linkam TP 94 microscopy hot stage for heating/cooling and annealing experiments. The Nanosensor silicon SPM probes sensors (type XYNCHR) had tip radii less than $10 \mathrm{~nm}$, the nominal spring constant and resonance frequency of the cantilever were respectively, $42 \mathrm{~N} / \mathrm{m}$ and $330 \mathrm{kHz}$. The height and phase contrast images were recorded with a set-point amplitude of $60-65 \%$ in order to minimize the interaction of the tip with the soft and sticky sample surface (soft tapping). A typical scan size was $12.5 \times 12.5 \mu \mathrm{m}$; the recording time per frame was approx 2 min. For the isothermal calorimetric experiment, a TA instruments DSC Discovery Series was used. The sample was heated to $120{ }^{\circ} \mathrm{C}$, held for 10 min and quenched to $60{ }^{\circ} \mathrm{C}$ and isothermally hold for $60 \mathrm{~min}$.

\section{Results}

Kinetics of polymer crystallization and or demixing of polymers in a blend are "classically" investigated by means of isothermal DSC $[14,15]$.

The performed isothermal calorimetric experiments however did not provide suitable data, a treatment of the data to extract crystallization kinetics as usually applied for polymer crystallization [14, 15] is not feasible due to the extremely small heat effects connected with the steric hardening and the different and widely spread time constants (see Fig. 1) for the occurring processes (minutes to weeks).

However, the development of the microstructures during an isothermal experiment can be followed by AFM. For AFM-experiments in a fast changing environment, quick scanning is essential to prevent 


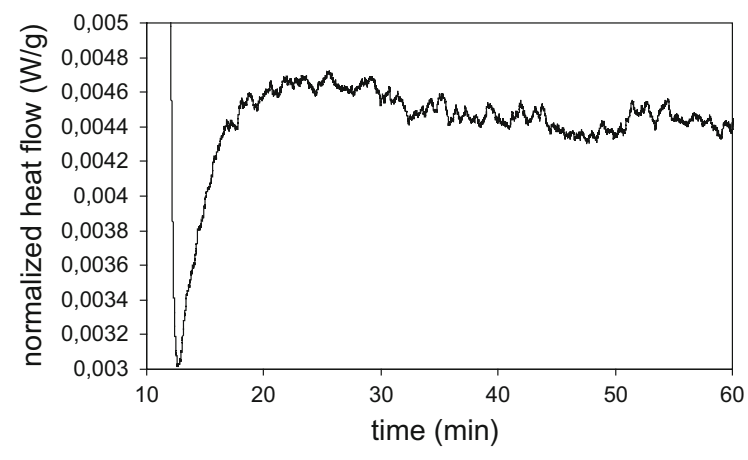

Fig. 1 Heat flow recorded during an isothermal DSC experiment of bitumen Q8 70-100 at $60{ }^{\circ} \mathrm{C}$ after quenching with ca. $30 \mathrm{~K} / \mathrm{min}$ from $120{ }^{\circ} \mathrm{C}$

creeping phenomena distorting the scanning during recording a snapshot of the crystallization process. The fastest scanning rate proved to provide acceptable images, which are applicable for studying crystallisation kinetics. The pictures obtained are subsequently linked to the isothermal DSC scan (see Fig. 2) and analyzed with respect to the distribution of surface area belonging to the catana/peri phase (i.e. the crystalline 'particle' phase) and to the perpetua phase (i.e. the more viscous 'matrix' phase).

The different stages of the process of physical ageing; nucleation and growth of the disperse phase (catana/peri-phase) as well as the diffusion and growth of its particles can be regarded as being similar to those processes occurring during crystallization in solutions which are supersaturated by cooling. Consequently, the kinetics of the formation of the equilibrium structures in bitumen can be treated within the framework of (heterogeneous) nucleation and crystallization theory.

The kinetics of isothermal crystallization of melts of low molecular compounds as well as of polymers can be described satisfactorily by the Avrami Eq. (1): $x_{\tau}=x_{\infty}\left(1-\mathrm{e}^{-k \tau^{n}}\right)$

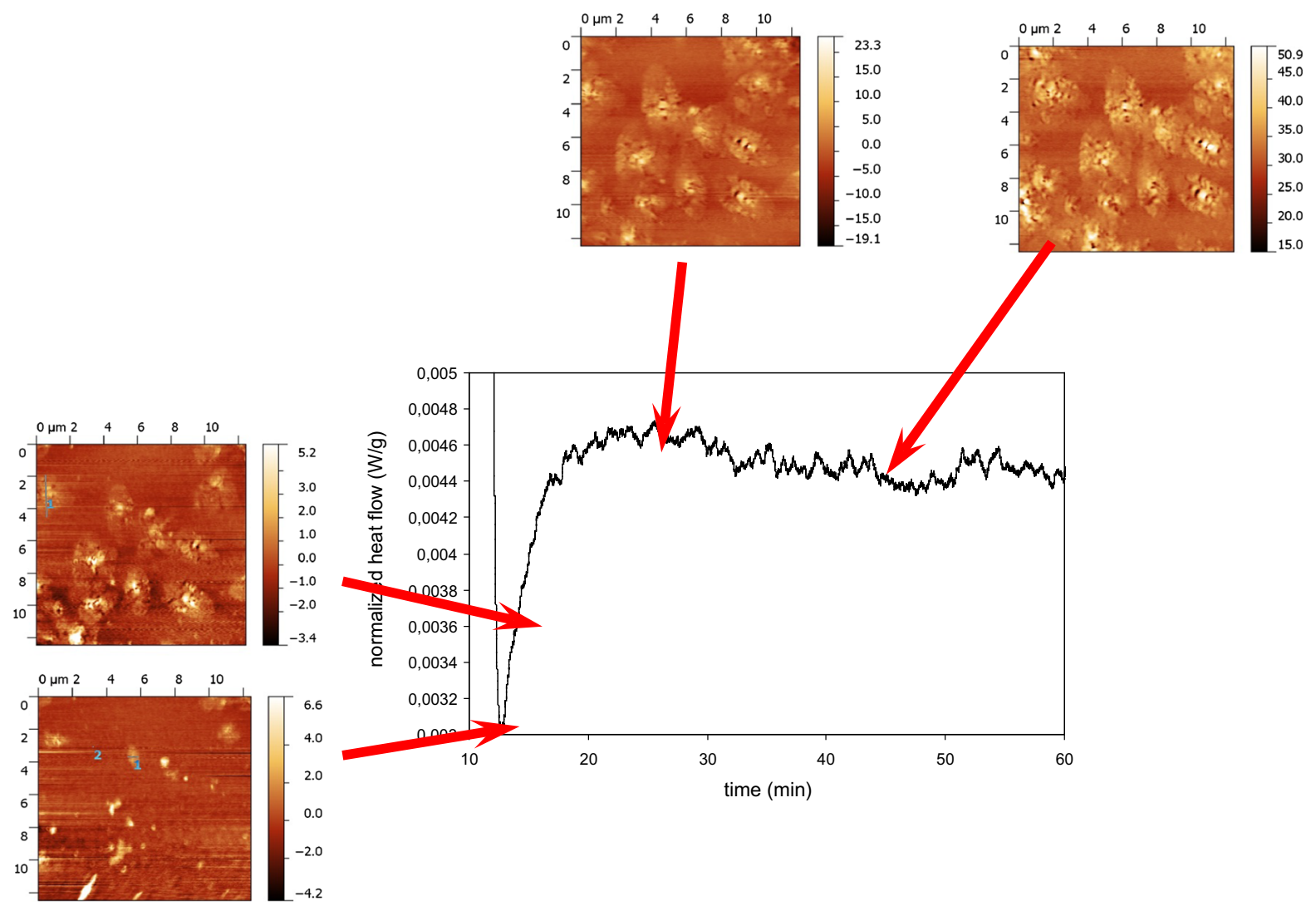

Fig. 2 Heat flow recorded during an isothermal DSC experiment of bitumen Q8 70-100 at $60{ }^{\circ} \mathrm{C}$ after quenching with ca. $30 \mathrm{~K} / \mathrm{min}$ from $120^{\circ} \mathrm{C}$ combined with the by AFM recorded phase contrast pictures 
With $x_{\tau}$ as the degree of crystallization at the time $\tau ; x_{\propto}$ as the degree of crystallization at the end of the studied crystallization process, $k$ as the crystallization rate constant; $n$ is a coefficient depending on the growth of the crystals (the Avrami exponent) and hence describing the mechanism of formation.

The experimentally determined value of the Avrami exponent allows assumptions concerning the mechanism of crystallization.

Following Lorenzo et al. [10], this exponent can be considered to be composed by two terms (2):

$n=n_{\mathrm{d}}+n_{\mathrm{n}}$

with $n_{\mathrm{d}}$ representing the dimensionality of the growing crystals (can only have integer values $1-3$ ) and $n_{\mathrm{n}}$ as the time dependency of nucleation (in case of homogeneous nucleation 1 and of heterogeneous nucleation 0 ).

However, since in some cases there is a mixed nucleation also non-integer values of the Avrami exponent may be obtained. The larger the value of $n$ is, the more perfectly the crystals are formed. For processes with n equal to 3 or 4 it is characteristic to find the growth of three-dimensional spherolites from instantaneously forming or gradually developing nuclei.

Previous investigations of crystallization kinetics of bitumen binders in bulk covering a time scale of days and using dilatometry resulted in a value of the Avrami parameter equal to 1 , independent on the composition or hardness (PEN-grade) of the bitumen samples [16]. It was observed, that the change in bitumen density occurred most rapidly at $50{ }^{\circ} \mathrm{C}$ i.e., at the temperature which is also characteristic for the maximum change in shear stress (softening). The growth of the dense structures was described to be controlled by diffusion processes in the system as characteristic of a crystallization process. However, the degree of order and the perfection of the particles of the new phase which was formed were not high. Complete crystallization was absent, the observed change in density was small (about $0.55 \%$ ) and the time constant was about $6 \mathrm{~h} \mathrm{[16].}$

The degree of crystallization $x_{\tau}$ can be determined from the ratio of peri-to perpetua-phase, while analyzing changes in dimensions of the forming and growing of crystals of the peri-phase.

Looking at the data obtained from AFM phase contrast pictures of the samples during the course of isothermal crystallization, firstly the fast development of flat, sheet-like crystals in time can be observed. These will be also the very first signs of crystallization since the temperature drop at the air interface will be the largest and the crystals formed by saturates display a lower density than the bulk bitumen (Fig. 4). However, since the overall degree of crystallization is rather small, there will be enough area for the crystals to form at the surface without interfering with each other.

The course of crystallization can be therefore described by using the development of relative area as registered by the phase contrast signal during scanning using the AFM instead of determining the volume of the growing crystalline phase in time, Eq. (3) can be applied:

$x_{\tau v}=\frac{V_{\tau}-V_{\infty}}{V-V_{\infty}} \sim x_{\tau s}=\frac{A_{\tau}-A_{\infty}}{A-A_{\infty}}$

with $x_{\tau v}$ as as the degree of crystallization in bulk (volume) and $x_{\tau s}$ as as the degree of crystallization as observable on the surface.

The shortest time to record a complete image was approx. $2 \mathrm{~min}$; pictures were taken immediately after cooling down (quenching) from $120{ }^{\circ} \mathrm{C}$ with a speed of $\geq 30 \mathrm{~K} / \mathrm{min}$ and reaching $60{ }^{\circ} \mathrm{C}$. The development of crystallinity (area of crystalline catana/peri phase) in time is plotted in Fig. 3.

The data obtained from the phase contrast images can be satisfactorily fitted using Eq. (1). Additionally, the kinetic constant can be calculated from the fit, a value of $k=5 \mathrm{~min}$. Also the half time of initial

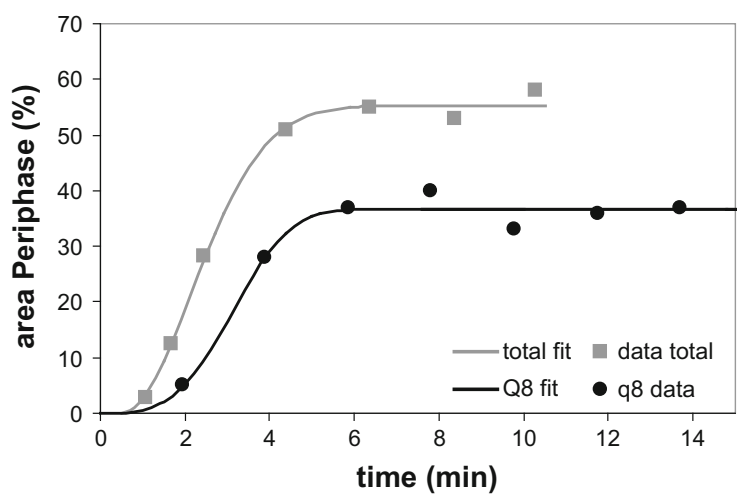

Fig. 3 Development of surface crystallinity in time and fit according to Eq. (1) 
crystallization $\tau_{1 / 2} \sim 3 \mathrm{~min}$. for the bitumen Q8 and $2 \min 25 \mathrm{~s}$ for the Total bitumen was found.

The variation in $n$-value (Avrami parameter during the isothermal crystallization can now be computed according to Eq. (4).

$n=\frac{-\tau\left(\frac{\mathrm{d} x_{\tau}}{\mathrm{d} \tau}\right)}{\left[\left(x_{\infty}-x_{\tau}\right) \ln \left(1-\frac{x_{\tau}}{x_{\infty}}\right)\right]}$

and is plotted in Fig. 4 together with schematical pictures for the evolution of crystallisation in time.

\section{Conclusions}

The variation in $n$ value with increasing relative crystallinity provides insights into the mechanism of crystallisation and the result with respect to crystal structure and perfection. It can be seen in Fig. 4 that the Avrami exponent changed progressively from 4 to 1.8 during the initial development of crystallinity with an average value of about 2.6.

Crystallisation starts almost immediately after or instantaneously during cooling. An earlier study by
Masson et al. [17]. proposed liquid liquid demixing within bitumen upon cooling resulting finally in steric hardening. The beginning of the crystallization is homogeneously nucleated and three-dimensional, which changes in the course of crystallization to 2-dimensional crystal growth and mixed nucleation, both homogeneous and heterogeneous. The primary crystallization process is very fast and followed by subsequent hardening events as reported by Pechenyi and Kusnetzov [16] and by Masson et al. [8, 17]. This especially the case in the Total bitumen sample, which crystallizes very fast and reaches also an initially higher degree of crystallinity compared to the Q8 sample. In this case, the originally homogenously 3 dimensionally nucleated crystals changes to 2-dimensional crystals, which grow in a very short time.

The analogous nature of the kinetics of formation of the equilibrium structures in bitumina and of the crystallization process makes it possible to regard both these processes as first order transitions. The equilibrium structures of the peri-phases should consequently be classified as semi-crystalline. The formation of the peri-phase is connected with an increase in stiffness/ hardness and in density. Consequently crystallization

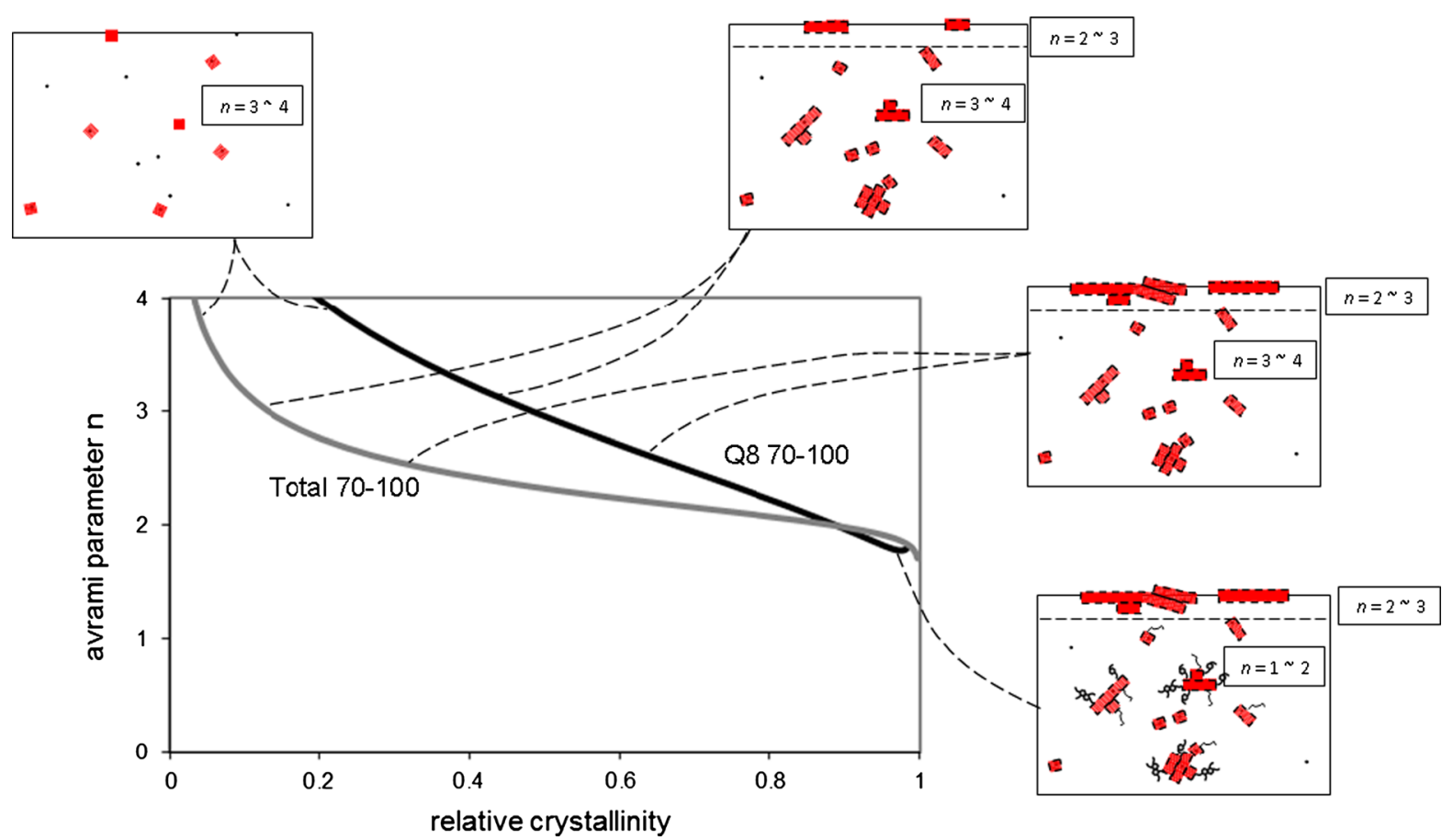

Fig. 4 Variation in $\mathrm{n}$ value during formation of the catana/peri phase at $60{ }^{\circ} \mathrm{C}$ and schematical pictures showing the evolution of crystallisation and of the crystal morphology/location of crystalline phase in time 
causes deformation creep and internal stresses, which promotes cracking.

\section{References}

1. Struik LCE (1978) Physical aging in amorphous polymers and other materials. Elsevier Scientific Publishing Company, New York

2. Traxler RN, Schweyer HE (1936) Increase in viscosity of asphalts with time. Proc Am Soc Test Mater 36(2):544-550

3. Traxler RN, Coombs CE (1937) Development of internal structure in asphalts with time. Proc Am Soc Test Mater 37(2):549-557

4. Nellensteyn F (1924) The constitution of asphalt. J Inst Pet Technol 10:311-323

5. Brown AB, Sparks JW, Smith FM (1957) Steric hardening of asphalts. Proc Assoc Asphalt Paving Technol 26:486-494

6. Blokker PC, Van Hoorn H (1959) Durability of bitumen in theory and practice. Proceedings of the 5th World Petroleum Congress, 417-432

7. Judycki J (2014) Influence of low-temperature physical hardening on stiffness and tensile strength of asphalt concrete and stone mastic asphalt. Constr Build Mater 61:191-199

8. Masson J-F, Polomark GM, Collins P (2002) Time-dependent microstructure of bitumen and its fractions by modulated differential scanning calorimetry. Energy Fuels $16: 470-476$

9. Togunde OP, Hesp SHM (1997) Physical hardening in asphalt mixtures. Int J Pavement Res Technol 5:46-53
10. Baglieri O, Dalmazzo D, Barazia M, Tabatabaee HA, Bahia HU (2012) Influence of physical hardening on the lowtemperature properties of bitumenand asphalt mixtures. SIIV - 5th International Congress-Sustainability of Road Infrastructures, Procedia-Social and Behavioral Sciences, vol 53, pp 504-513

11. Bahia HU, Anderson DA (1992) Physical hardening of paving grade asphalts as related to compositional characteristics. In: Preprints of Papers, 204th ACS National Meeting. Division of Fuel Chemistry, vol 37, No 4, American Chemical Society, Washington, DC, pp 1397-1407

12. Tabatabaee HA, Velasquez R, Bahia HU (2012) Predicting low temperature physical hardening in asphalt binders. Constr Build Mater 34:162-169

13. Fischer HR, Dillingh EC, Hermse CGM (2014) On the microstructure of bituminous binders. Road Mat Pavem Design 15:1-15

14. Lorenzo AT, Arnal MA, Albuerne J, Müller AJ (2007) DSC isothermal polymer crystallization kinetics measurements and the use of the Avrami equation to fit the data: guidelines to avoid common problems. Polym Test 26:222-231

15. Lu XF, Hay JN (2001) Isothermal crystallization kinetics and melting behaviour of poly(ethylene terephthalate). Polymer 42(23):9423-9431

16. Pechenyi BG, Kuznetsov OI (1990) Formation of equilibrium structures in bitumens. Chem Technol Fuels Oils 26(7):372-376 (Translated From Khimiya i Tekhnologiya Topliv i Masel, 7, 32-34)

17. Masson J-F, Collins P, Polomark G (2005) Steric hardening and the ordering of asphaltenes in bitumen. Energy Fuels 19(1):120-122 